\title{
Toward stable 3D numerical evolutions of black-hole spacetimes
}

\author{
Mark A. Scheel, ${ }^{1}$ Lawrence E. Kidder, ${ }^{2}$ Lee Lindblom, ${ }^{1}$ Harald P. Pfeiffer, ${ }^{2}$ and Saul A. Teukolsky ${ }^{2}$ \\ ${ }^{1}$ Theoretical Astrophysics 130-33, California Institute of Technology, Pasadena, California 91125 \\ ${ }^{2}$ Center for Radiophysics and Space Research, Cornell University, Ithaca, New York 14853
}

(Received 30 September 2002; published 17 December 2002)

\begin{abstract}
Three dimensional (3D) numerical evolutions of static black holes with excision are presented. These evolutions extend to about $8000 M$, where $M$ is the mass of the black hole. This degree of stability is achieved by using growth-rate estimates to guide the fine tuning of the parameters in a multiparameter family of symmetric hyperbolic representations of the Einstein evolution equations. These evolutions were performed using a fixed gauge in order to separate the intrinsic stability of the evolution equations from the effects of stability-enhancing gauge choices.
\end{abstract}

DOI: 10.1103/PhysRevD.66.124005

PACS number(s): 04.25.Dm, 02.30.Mv, 02.60.Cb, 04.20.Ex

Recent studies have documented the fact that constraintviolating instabilities are a common (if not universal) feature of solutions to the Einstein evolution equations [1-3]. Initial data with small numerical errors on some initial Cauchy surface will typically evolve to a solution in which the constraints grow exponentially with time. Black-hole spacetimes that are evolved in full 3D (without symmetry) with a fixed gauge using one of the "standard" formulations of the evolution equations [e.g. Arnowitt-Deser-Misner (ADM) [4,5] or Baumgarte-Shapiro-Shibata-Nakamura (BSSN) [6,7]] have instabilities of this type that become unphysical (e.g. because the constraints become large) on a time scale of about $100 \mathrm{M}$ $[8,9]$, where $M$ is the mass of the black hole. Several studies have shown that changing the evolution equations by adding multiples of the constraints and by changing the dynamical fields can have a significant effect on the growth rate of these constraint-violating instabilities [1-3]. Such a reformulation of the BSSN evolution equations has allowed full 3D evolutions with fixed gauge to persist for about 1400M [10]. The duration of black hole evolutions has also been extended considerably, apparently indefinitely in some cases, by imposing symmetries, e.g. octant, on the solutions [11] or by using an appropriate dynamical gauge $[8,12]$.

We present new results for evolving isolated static black holes using a multiparameter family of symmetric hyperbolic representations of the Einstein evolution equations [1]. For the optimal case our evolutions extend to about $8000 M$. We focus on the question of how the evolution equations themselves affect stability, and therefore we use a fixed gauge [20] and do not impose any symmetries on the solutions. The fine tuning needed to achieve optimal stability for evolving a single black hole requires a special choice of the parameters in our representation of the evolution equations, but does not require any fine tuning of our numerical methods. Thus we expect that any numerically stable evolution code that solves this same system of equations with the same initial data and boundary conditions will exhibit the same behavior we find here.

We study the evolution of black-hole spacetimes using a particular 12-parameter family of representations of the Einstein evolution equations [1]. This family is derived from the standard 3+1 "ADM" form of the equations by introducing five parameters $\{\gamma, \zeta, \eta, \chi, \sigma\}$ that densitize the lapse func- tion and add multiples of the constraints to the evolution equations, and seven additional parameters $\{\hat{z}, \hat{k}, \hat{a}, \hat{b}, \hat{c}, \hat{d}, \hat{e}\}$ that redefine the set of dynamical fields. The details of the resulting evolution equations and the precise definitions of these various parameters are explained at length elsewhere $[1,3]$, and will not be repeated here. It has been shown that a 9-parameter subfamily of these representations consists of strongly hyperbolic evolution equations in which all of the characteristic speeds of the system (relative to the hypersurface-normal observers) have only the physical values: $\{0, \pm 1\}[1]$. It has also been shown that the evolution equations for an open subset of this 9-parameter family, in particular those representations with $-\frac{5}{3}<\zeta<0$, are symmetric hyperbolic [3]. Our numerical analysis here is confined to this 9-parameter family of symmetric hyperbolic representations of the Einstein evolution equations having physical characteristic speeds.

Here we analyze the numerical evolution of initial data that represents a single isolated static black hole. For initial data we use a $t=$ constant slice of the Schwarzschild geometry written in Painlevé-Gullstrand coordinates [13],

$$
d s^{2}=-d t^{2}+\left(d r+\sqrt{\frac{2 M}{r}} d t\right)^{2}+r^{2} d \Omega^{2}
$$

(where $d \Omega^{2}$ is the standard metric on the unit sphere), plus small perturbations that are added by hand. By explicitly inserting the same perturbations for all numerical resolutions, we are able to test convergence; this would not be the case if instead we allowed the perturbations to arise from machine roundoff error. The exact form of the perturbations is unimportant; it does not affect either the asymptotic growth rate of the unstable mode or its spatial dependence.

We also fix the gauge for these evolutions (not just at the initial time but throughout the evolution) by setting the densitized lapse and the shift to those of Eq. (1). Fixing the gauge in this way is known to be less stable than using a carefully selected dynamically determined gauge $[8,9]$. However, our purpose here is to study the intrinsic stability of the evolution equations, so we choose to fix the gauge in this nonoptimal way in order to isolate and emphasize this instability. 


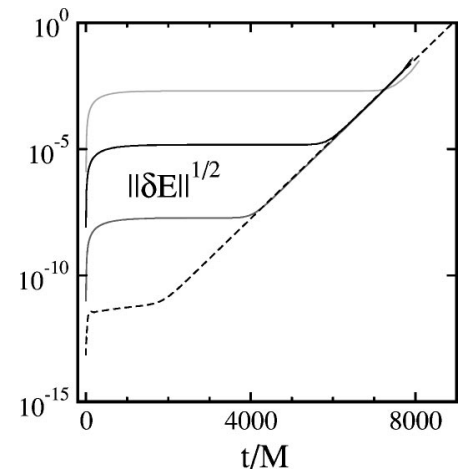

FIG. 1. Energy norm $\|\delta E\|^{1 / 2}$ (per unit volume) for the most stable set of evolution parameters. Solid curves are $\|\delta E\|^{1 / 2}$ from the full nonlinear evolution code, and the dashed curve is from a linearized version of the code.

The evolution equations are solved here using a pseudospectral collocation method (see $[1,14,15]$ for further details on the implementation) on a 3D spherical shell extending (typically) from $r=1.9 M$ to $r=11.9 M$. This code utilizes the method of lines; the time integration is performed using a fourth-order Runge-Kutta algorithm. Although we use spherical coordinates, our fundamental variables are the Cartesian components of the various fields. The inner boundary lies inside the event horizon; at this boundary all the characteristic curves are directed out of the domain (into the black hole), so no boundary condition is required there and none is imposed ("horizon excision"). At the outer boundary we require that all ingoing characteristic fields be timeindependent, but we allow all outgoing characteristic fields to propagate freely.

Recent analytical work [3] has shown that the growth rates of the constraint-violating instabilities for the PainlevéGullstrand form of the Schwarzschild geometry depend on just three of the nine parameters that specify the evolution equations, $\{\gamma, \zeta, \hat{z}\}$. We confine our study here to the dependence of this instability on the two parameters $\{\gamma, \hat{z}\}[21]$, and we fix the remaining parameters to the values that define system III of Ref. [1].

Figure 1 shows numerical results from the evolution of a single black hole for the case $\gamma=-12, \hat{z}=-0.425$. Plotted is the energy norm $\|\delta E\|^{1 / 2}$ (as introduced in Ref. [3]), which measures the deviation of the numerical solution from an exact solution that satisfies the constraints. The solid curves in Fig. 1 represent computations performed at different spectral resolutions (18, 24, and 32 radial collocation points), and thus illustrate the convergence of our solutions. The dashed curve represents the evolution obtained with a linearized version of the code, normalized so that the amplitude of the unstable mode is the same as that obtained with the nonlinear evolution. The convergence of these solutions, as illustrated in Fig. 1, is made possible by choosing the same initial data, including the exact same form for the initial perturbation added by hand to Eq. (1), for each resolution. If we had instead chosen initial data given by Eq. (1) plus random perturbations (either supplied by numerical roundoff error or introduced by hand) we would not expect results using different resolutions to converge to the same solution.

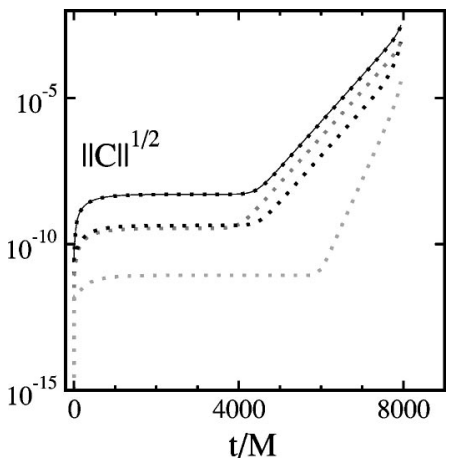

FIG. 2. Solid curve shows the evolution of the integral norm of all the constraints $\left\|\mathcal{C}_{k i j} \mathcal{C}^{k i j}+\mathcal{C}_{k} \mathcal{C}^{k}+\mathcal{C}_{k l i j} \mathcal{C}^{k l i j}+\mathcal{C}^{2}\right\|^{1 / 2}$ (per unit volume) for the most stable set of evolution parameters. Dotted curves show the individual contributions from the various constraints: $\mathcal{C}_{k i j} \mathcal{C}^{k i j}, \mathcal{C}_{k l i j} \mathcal{C}^{k l i j}, \mathcal{C}_{k} \mathcal{C}^{k}$ and $\mathcal{C}^{2}$ (in that order from largest to smallest at late times).

Figure 2 shows the evolution of the integral norm of the constraints (see Refs. [1,3] for definitions of the constraint variables) for the highest-resolution case shown in Fig. 1. Note that at late times, most of the constraints in Fig. 2 grow at the same rate $(1 / \tau \approx 1 / 275 M)$ as the energy norm shown in Fig. 1. The exception is the Hamiltonian constraint, which is much smaller than the other constraints, but grows at double the growth rate, $1 / \tau \approx 1 / 137 M$. Thus it appears that for the optimal choice of parameters, the unstable mode violates the Hamiltonian constraint only to second order in the mode amplitude.

Given a numerical evolution for a particular set of parameters, we determine the exponential growth rate by measuring the slope of the curve in Figs. 1 or 2. Figures 3 and 4 illustrate these growth rates as functions of the parameters $\gamma$ and $\hat{z}$. The points in these figures represent numerically determined growth rates measured using the linearized code (which yields the same growth rates as the fully nonlinear code; see Fig. 1 and Ref. [3]). The solid curves represent the simple a priori estimates of these growth rates introduced in Ref. [3]. Although the agreement between the estimates and the numerical results is only approximate, this agreement was good enough to allow us to direct our search for the most stable values of the parameters to the relevant region of the parameter space. The curves in these figures represent

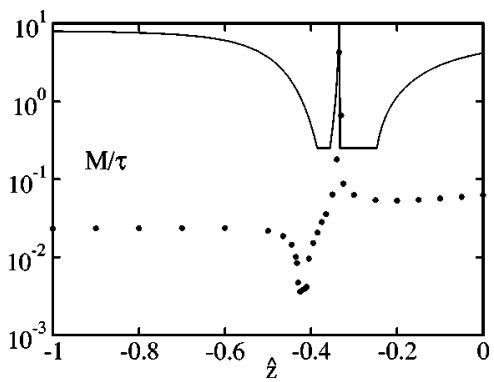

FIG. 3. Exponential growth rates of the constraint-violating instabilities as a function of the parameter $\hat{z}$ (with fixed $\gamma=-12$ ). Points are numerically determined rates, while the solid curve is the approximate growth rate. 


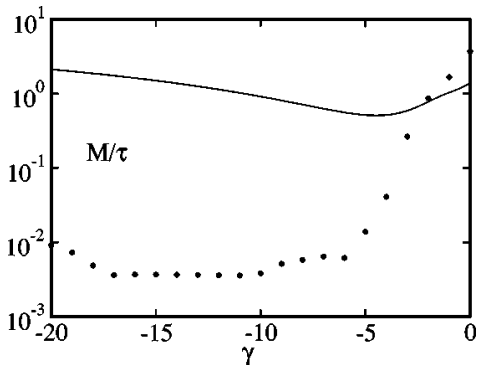

FIG. 4. Exponential growth rates of the constraint-violating instabilities as a function of the parameter $\gamma$ (with fixed $\hat{z}$ $=-0.425)$. Points are numerically determined rates, while the solid curve is the approximate growth rate.

orthogonal slices of the function $1 / \tau(\gamma, \hat{z})$ through its minimum, $1 / \tau_{\max }=1 / 275 M$, which occurs at the parameter values $\gamma=-12$ and $\hat{z}=-0.425$. This minimum growth rate is such that constraint violations in the initial data that are comparable to typical machine precision (e.g. $10^{-16}$ ) will become large (e.g. of order 0.1 ) when $t \approx 10^{4} M$. Figures 1 and 2 illustrate the full nonlinear evolution that corresponds to this optimal choice of parameters.

For all the cases discussed so far, the outer boundary radius was set at $r_{\max }=11.9 M$. Figure 5 illustrates the dependence of the growth rate $1 / \tau$ on the location of the outer boundary of our computational domain, for fixed $\gamma=-12$ and $\hat{z}=-0.425$. This curve shows a sharp local minimum at the radius where the optimal set of evolution parameters $\{\gamma, \hat{z}\}$ was determined, strongly suggesting that these optimal values depend on the location of this outer boundary. We have verified this by studying in some detail the case where the outer boundary is located at $r_{\max }=81.9 \mathrm{M}$. There we find that the new optimal values of the parameters become $\gamma$ $=-12$ and $\hat{z}=-0.41$, and the value of the growth rate at these new optimal parameters becomes $1 / \tau=1 / 333 M$. This growth rate is about $20 \%$ smaller than that of the system whose evolution is illustrated in Fig. 1. Thus we infer that the evolution of a single black hole in this case would extend to about $10^{4} \mathrm{M}$. We also note that the optimal parameters for $r_{\max }=81.9 M$ give a value of $1 / \tau$ that is about $2 / 3$ the value illustrated in Fig. 5 for this value of $r_{\max }$. Considerable additional computational effort will be required to determine the general dependence of the optimal value of $1 / \tau$ on $r_{\max }$, and we postpone that to a future study. For $r_{\max }<12 M$ and

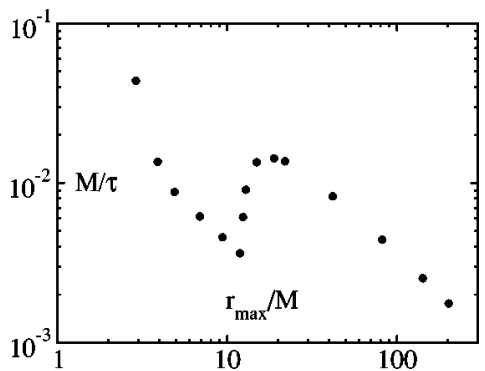

FIG. 5. Instability growth rates as a function of the location of the outer boundary of the computational domain for the evolution parameter values $\gamma=-12, \hat{z}=-0.425$.

for $r_{\max }>20 M$, the growth rate for a fixed set of evolution parameters decreases roughly like $\Lambda / r_{\max }$, with the constant $\Lambda$ being about a factor of six larger for the case with $r_{\max }$ $>20 M$. However, the optimal value of $1 / \tau$ as a function of $r_{\max }$ does not scale in this simple way. The smallest growth rate determined in our study to date is the point at $\gamma=-12$ and $\hat{z}=-0.425$ with $r_{\max }=201.9 M$, where we find $1 / \tau$ $=1 / 570 M$. This evolution would be expected to persist for over $16000 M$.

Finally, we note that all of the numerical evolutions discussed so far have placed the inner boundary of the domain at $r_{\min }=1.9 \mathrm{M}$. We have also run the code with $r_{\min }=1.0 \mathrm{M}$ and $r_{\min }=1.5 \mathrm{M}$ for our best-studied case $(\gamma=-12, \hat{z}$ $\left.=-0.425, r_{\max }=11.9 M\right)$ and we find that the growth rate is the same to three significant digits.

In summary, we have illustrated that significant improvements in the stability of numerical evolutions of 3D blackhole spacetimes can be achieved by a careful choice of the representation of the Einstein evolution equations. In particular we have shown that single black hole spacetimes can be evolved longer than $t \approx 8000 M$ even with fixed gauge. These new results also indicate that the outer boundary conditions may play a significant role in fixing the optimal formulation of the equations, as has been suggested by other investigations [16-19]. The role of these boundary conditions will be explored more thoroughly in a future study.

Some computations were performed on the IA-32 Linux cluster at NCSA. This research was supported in part by NSF grant PHY-0099568 and NASA grant NAG5-10707 at California Institute of Technology and NSF grants PHY-9800737 and PHY-9900672 at Cornell University.
[1] L.E. Kidder, M.A. Scheel, and S.A. Teukolsky, Phys. Rev. D 64, 064017 (2001).

[2] P. Laguna and D. Shoemaker, Class. Quantum Grav. 19, 3679 (2002).

[3] L. Lindblom and M.A. Scheel, Phys. Rev. D 66, 084014 (2002).

[4] R. Arnowitt, S. Deser, and C.W. Misner, in Gravitation: An Introduction to Current Research, edited by L. Witten (Wiley, New York, 1962), pp. 227-265.

[5] J.W. York, Jr., in Sources of Gravitational Radiation, edited by
L.L. Smarr (Cambridge University Press, Cambridge, England, 1979), pp. 83-126.

[6] M. Shibata and T. Nakamura, Phys. Rev. D 52, 5428 (1995).

[7] T.W. Baumgarte and S.L. Shapiro, Phys. Rev. D 59, 024007 (1999).

[8] H.-J. Yo, T.W. Baumgarte, and S.L. Shapiro, Phys. Rev. D 66, 084026 (2002).

[9] M. Alcubierre, B. Brügmann, T. Dramlitsch, J.A. Font, P. Papadopoulos, E. Seidel, N. Stergioulas, and R. Takahashi, Phys. Rev. D 62, 044034 (2000). 
[10] D. Shoemaker, K. Smith, E. Schnetter, D. Fiske, and P. Laguna, "Maya: A Black Hole Evolution Code" (in preparation).

[11] M. Alcubierre and B. Brügmann, Phys. Rev. D 63, 104006 (2001).

[12] M. Alcubierre, B. Brügmann, P. Diener, M. Koppitz, D. Pollney, E. Seidel, and R. Takahashi, "Gauge conditions for longterm numerical black hole evolutions without excision," gr-qc/0206072.

[13] K. Martel and E. Poisson, Am. J. Phys. 69, 476 (2001).

[14] L.E. Kidder, M.A. Scheel, S.A. Teukolsky, E.D. Carlson, and G.B. Cook, Phys. Rev. D 62, 084032 (2000).

[15] L.E. Kidder, M.A. Scheel, H.P. Pfeiffer, and S.A. Teukolsky (in preparation).

[16] H. Friedrich and G. Nagy, Commun. Math. Phys. 201, 619
(1999).

[17] G. Calabrese, L. Lehner, and M. Tiglio, Phys. Rev. D 65, 104031 (2002).

[18] B. Szilágyi and J. Winicour, "Well-posed initial-boundary evolution in general relativity," gr-qc/0205044.

[19] G. Calabrese, J. Pullin, O. Sarbach, M. Tiglio, and O. Reula, "Well posed constraint-preserving boundary conditions for the linearized Einstein equations," gr-qc/0209017.

[20] We use a fixed densitized lapse and fixed shift. For the evolution equations studied here fixing the lapse itself violates hyperbolicity, which is inconsistent with the way our code imposes boundary conditions.

[21] The analytical estimates [3] suggest that varying the third parameter $\zeta$ should not result in significantly increased stability. 